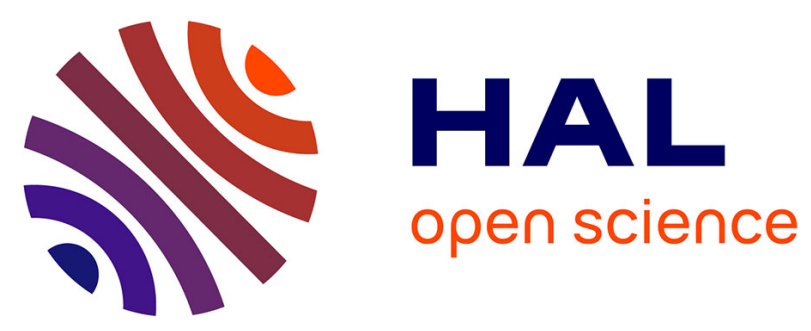

\title{
Rodent : Intronic E-box sequences control tissue specificity but not rhythmic expression in the pineal gland
}

Ann Humphries, Tim Wells, Ruben Baler, David C. Klein, David A. Carter

\section{- To cite this version:}

Ann Humphries, Tim Wells, Ruben Baler, David C. Klein, David A. Carter. Rodent: Intronic E-box sequences control tissue specificity but not rhythmic expression in the pineal gland. Molecular and Cellular Endocrinology, 2007, 270 (1-2), pp.43. 10.1016/j.mce.2007.02.003 . hal-00531915

\section{HAL Id: hal-00531915 https://hal.science/hal-00531915}

Submitted on 4 Nov 2010

HAL is a multi-disciplinary open access archive for the deposit and dissemination of scientific research documents, whether they are published or not. The documents may come from teaching and research institutions in France or abroad, or from public or private research centers.
L'archive ouverte pluridisciplinaire HAL, est destinée au dépôt et à la diffusion de documents scientifiques de niveau recherche, publiés ou non, émanant des établissements d'enseignement et de recherche français ou étrangers, des laboratoires publics ou privés. 


\section{Accepted Manuscript}

Title: Rodent Aanat: Intronic E-box sequences control tissue specificity but not rhythmic expression in the pineal gland

Authors: Ann Humphries, Tim Wells, Ruben Baler, David C. Klein, David A. Carter

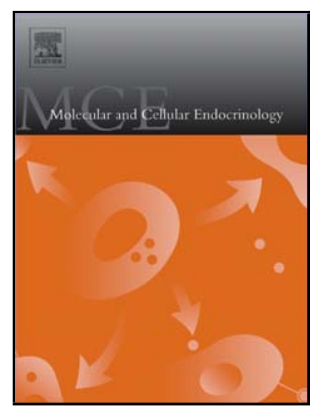

PII:

DOI:

Reference:

To appear in:

Received date:

Revised date:

Accepted date:
S0303-7207(07)00079-2

doi:10.1016/j.mce.2007.02.003

MCE 6623

Molecular and Cellular Endocrinology

$30-12-2006$

$7-2-2007$

$7-2-2007$

Please cite this article as: Humphries, A., Wells, T., Baler, R., Klein, D.C., Carter, D.A., Rodent Aanat: Intronic E-box sequences control tissue specificity but not rhythmic expression in the pineal gland, Molecular and Cellular Endocrinology (2007), doi:10.1016/j.mce.2007.02.003

This is a PDF file of an unedited manuscript that has been accepted for publication. As a service to our customers we are providing this early version of the manuscript. The manuscript will undergo copyediting, typesetting, and review of the resulting proof before it is published in its final form. Please note that during the production process errors may be discovered which could affect the content, and all legal disclaimers that apply to the journal pertain. 
Rodent Aanat: Intronic E-box sequences control tissue specificity but not rhythmic expression in the pineal gland.

Ann Humphries ${ }^{1}$, Tim Wells ${ }^{1}$, Ruben Baler ${ }^{2}$, David C. Klein ${ }^{3}$, David A. Carter ${ }^{1}$.

${ }^{1}$ School of Biosciences, Cardiff University, Cardiff, UK, ${ }^{2}$ Science Policy Branch, National Institute on Drug Abuse, ${ }^{3}$ Section on Neuroendocrinology, Office of the Scientific Director, National Institute of Child Health and Human Development, National Institutes of Health, Bethesda, MD 20892, USA.

Correspondence:

Dr.D.A.Carter,

School of Biosciences,

Cardiff University,

PO BOX 911, Museum Avenue,

Cardiff CF10 3US. UK.

Tel: 02920874095

Fax: 02920876328

Email:smbdac@cardiff.ac.uk 
KEY WORDS: Arylalkylamine $N$-acetyltransferase; pineal gland; transgenic rat; E-box; circadian rhythm.

\begin{abstract}
Arylalkylamine $\mathrm{N}$-acetyltransferase (Aanat) is the penultimate enzyme in the serotonin $\mathrm{N}$-acetylserotonin - melatonin pathway. It is nearly exclusively expressed in the pineal gland and the retina. A marked rhythm of Aanat gene expression in the rat pineal is mediated by cyclic AMP response elements located in the promoter and first intron. Intron 1 also contains E-box elements, which mediate circadian gene expression in other cells. Here we examined whether these elements contribute to rhythmic Aanat expression in the pineal gland. This was done using transgenic rats carrying Aanat transgenes with mutant E-box elements. Circadian expression of Aanat transgenes was not altered by these mutations. However, these mutations enhanced ectopic expression establishing that the intronic Aanat E-box elements contribute to the gene's pineal specific expression. A similar role of the Aanat E-box has been reported in zebrafish, indicating that Aanat Ebox mediated silencing is a conserved feature of vertebrate biology.
\end{abstract}




\section{INTRODUCTION}

Daily rhythms of melatonin production in the pineal gland are governed by a rhythm in the synthesis and activity by the penultimate enzyme in the melatonin pathway, arylalkylamine N-acetyltransferase, Aanat; Coon et al, 1995; Klein et al, 1997; Klein, 2006). This enzyme is encoded by Aanat which is rhythmically expressed in the rodent pineal gland, resulting in a $\sim 150$-fold increase in the abundance of Aanat mRNA at night (Coon et al., 1995; Roseboom et al, 1996; Klein 1997).

The increase in expression of the Aanat gene in the rat pineal gland appears to result from cis-acting mechanisms that involve a cAMP-responsive element (CRE)-CCAAT complex within the proximal promoter and a CRE located in the first intron (Baler et al, 1997;

Baler et al., 1999). Transcription appears to be activated in response to cAMP-dependent phosphorylation of CRE binding protein (CREB) (Roseboom et al, 1995; Maronde et al, 1999). The first intron of the rat Aanat gene also contains an E-box element (Baler et al, 1999; Fig.1A), which is of interest because a sub-set of E-boxes, described as circadian E-boxes (Munoz et al, 2002) mediate rhythmic gene expression through interaction with the trans-acting clock proteins CLOCK and BMA11 (Jin et al, 1999; Reppert \& Weaver, 2002); clock and Bmall are both expressed in the rat pineal gland (Namahira et al, 1999). Furthermore, functional Aanat E-box elements have been characterized in other species (Appelbaum et al, 2004; Chong et al, 2000); in the zebrafish these elements appear to form part of an enhancer (PRDM, pineal-restrictive downstream module) that includes photoreceptor conserved elements (PCEs). PCEs are found in genes expressed in the pineal gland and retina and are thought to be essential for this pattern of expression 
(Appelbaum and Gothilf, 2006). This enhancer contributes not only to the control of rhythmic expression of Aanat but also to the determination of tissue-specific zebrafish (zf) Aanat expression; the mechanism appears to reflect the interaction of OTX proteins and PCE in the enhancer (Appelbaum et al, 2005).

The $\sim 2 \mathrm{~kb}$ region between -218 and +1786 (relative to transcription start site) in the rat Aanat gene is sufficient to mediate both rhythmic and tissue-specific expression of rat Aanat (Burke et al, 1999; Smith et al, 2001). Of special interest is the importance of intron I to the rhythmic expression of Aanat. Little is known about this region, other than gross deletion results in decreased pineal expression and increased ectopic expression (Burke et al, 1999) and that it appears to influence the basal level of expression of the gene in non-pineal cells; in addition the CRE in intron 1 amplifies in vitro cAMP responsiveness of reporter constructs containing the CRE/CCAAT complex (Baler et al, 1999). The role of other putative regulatory elements in intron 1 has not been determined.

Here, we examined the role of two related E-box elements in intron 1, a consensus E-box element (CACGTG) and an E-box-like element (CACATG) (Figure 1A). This was done using a reporter transgene composed of the $\sim 2 \mathrm{~kb}$ sequence that contains the promoter region and intron 1 of Aanat described above. The role of the intronic E-box elements was examined in vivo in animals carrying a reporter construct in which these elements were distrupted by the insertion of one or two bases. The results of these studies are presented here. 


\section{METHODS}

Design of constructs and transgenic rat production. The Aanat-luciferase transgene construct used in the production of the novel lines of transgenic rats reported here has been described (Burke et al, 1999). This transgene (-218/Luc, which served as the wildtype control for the current study, contains Aanat genomic sequence from position -218 to +1786 relative to the transcription start site, and includes the entire 1612 nucleotide intron 1 sequence (Fig.1 A, 1B). A mutant E-box transgene $(-218 \mathrm{mEB} / \mathrm{Luc}$ was derived from the wild-type transgene by introducing three point mutations: the consensus E-box at position +623 was changed from CACGTG to CACAGTG and the E-box like sequence at position +636 was changed from CACATG to CACTTATG (Fig.1B; see Chen et al, 2000). These sequences were selected for mutation firstly because the $+623 \mathrm{E}-$ box is the only consensus E-box within intron 1, and secondly because both this E-box and the +636 E-box-like sequence are both fully conserved between rat and mouse, lying within a $24 \mathrm{bp}$ run of intronic sequence that is $100 \%$ conserved between these rodent genomes (Ensembl genes: ENSRNOG00000011182 and ENSMUSG00000020804). In contrast, other E-box-like CACATG sequences within intron 1 of both the rat $(+221$ and $+1486)$ and mouse $(+1336$ and +1354$)$ genomes are not located within highly conserved sequence. To confirm that the mutant sequences had been properly incorporated into the genome of transgenic rats, genomic DNA was isolated from tail biopsies and subjected to DNA sequencing using the Prism ${ }^{\mathrm{TM}}$ Ready Reaction Dye-Deoxy Terminator Cycle 
Sequencing Kit (Perkin-Elmer, Foster City, CA, USA) and an ABI Prism ${ }^{\mathrm{TM}}$ automated DNA sequencer (377; Perkin-Elmer).

Transgenic rats were generated as described (Burke et al, 1999). The genotype of individual transgenic founder animals was characterized by Southern blot analysis of PstI-digested genomic DNA using a 1.6kb NcoI/XbaI, luciferase-specfic fragment of the transgene (Fig.1B). Following the characterization of founders, multiple distinct lines of transgenics were established: three lines harboring the mutant E-box transgene (U20, U30, U42), and one line harbouring the wild-type transgene (W21).

Animals and sampling. Animal studies were conducted in accordance with both UK Home Office regulations, and local ethical review. Adult (3-4 month) Sprague-Dawley (CD) transgenic rats were maintained in standard laboratory conditions in a 14:10 light:dark cycle (lights on: $05.00 \mathrm{~h}$ ), and then transferred to constant darkness for a period of three days prior to sampling. Animals were killed by cervical dislocation at the indicated times of the daily cycle; tissues were rapidly dissected, and frozen on dry ice and stored.

qRPA analysis of transcript rhythms. Quantitative ribonuclease protection assays (qRPA) of gene rhythms were conducted using the RPA III kit according to the manufacturers instructions (Ambion, Austin, TX). qRPA probes were constructed by annealing and ligating pairs of complementary oligonucleotides into MultiProbe vectors: GAPDH, vector 2; AANAT, vector 6; luciferase, vector 9 (Ambion). Oligonucleotide 
sequences are provided in Table 1. The assay was designed according to the Ambion qRPA guidelines. Individual assays were conducted for each transcript. Assays were conducted on triplicate biological replicates (each replicate containing two pooled pineal glands) sampled from each of the four individual transgenic lines (U20, U30, U42, W21). For each time point, $0.5 \mathrm{mg}$ of total pineal RNA was used. Protected fragments were resolved on $10 \%$ TBE-Urea polyacrylamide gels and exposed to a Storage phosphor screen (Kodak K, Eastman Kodak, Rochester, MN, USA). The abundance of protected fragments was quantified by densitometry (ImageQuant ${ }^{\mathrm{TM}} 3.0$, GE Healthcare, Chalfont St. Giles, Bucks, UK), and Aanat and transgene transcript levels were corrected against levels of GAPDH mRNA. Statistical comparison of gene rhythms between wild-type and mutant transgenic lines was conducted by determining area under curve (AUC) of individual time courses (GraphPad Prism, 2.01, GraphPad Software Inc, Calabas, CA, USA), and then comparing groups of AUC values with independent samples Student's t tests (SPSS 13, SPSS Inc., Chicago, IL,USA).

Northern blot analysis of tissue expression. Total RNA was extracted and Northern blot analysis of tissue expression was conducted as described previously (Burke et al, 1999). The hypothalamic and cortical brain samples were obtained from a coronal brain slice as described in the dissection protocol of Glowinski and Iversen (1966). The sample of neocortex was obtained by cutting a $5 \mathrm{~mm}$ block through cortical layers I-VI of the right hemisphere at the level of the somatosensory cortex. Whole pituitary glands including both the adenohypophysis and neurointermediate lobe constituted the pituitary sample. The luciferase-specific probe (see above) was used to detect transgene transcripts and a 
truncated Aanat cDNA probe (Smith et al, 2001) used to specifically detect Aanat transcripts. Northern blots were stripped (boiling 0.1\% SDS, 3-5 x 2 min) and re-probed with a commercially available $18 \mathrm{~S}$ cDNA (DecaTemplate ${ }^{\mathrm{TM}}$, Ambion, Austin, Tx, USA). Assays were conducted on duplicate biological samples from three transgenic lines: U30, U42, W21. Densitometric comparison of mRNA levels between tissue samples was performed using ImageQuant ${ }^{\mathrm{TM}} 3.0$ (Amersham Pharmacia Biotech), correcting values against the corresponding level of 18S RNA.

In situ hybridization analysis of retinal transgene expression. In situ hybridization (ISH) analysis of retinal transgene expression was conducted on fresh frozen sections using standard non-radioactive protocols as described: (http://wwwetb.info.nih.gov/lcmr/snge/Protocols/ISHH/ISHH.html) using sense and antisense orientation RNA probes corresponding to bases $1737-2141$ of the pGL3 luciferase vector (Promega, Madison, WI). ISH analysis was conducted on multiple $12 \mu \mathrm{m}$ sections from each of the U30, U42, and W21 transgenic lines. Retinal sections were viewed under bright field optics (Leica DM-LB microscope) and images were captured using a Leica DFC-300FX digital camera, and Leica QWin software (V3; Leica Microsystems Imaging Solutions Ltd, Cambridge, UK).

\section{RESULTS}

Circadian expression. A marked circadian rhythm was detected in the expression of the wild-type transgene with transcripts being minimal during subjective day $(08.00 \mathrm{~h}-$ $18.00 \mathrm{~h})$ and intensely expressed during the middle period of subjective night $(22.00 \mathrm{~h}-$ 
03.00h). For the purposes of the present study we therefore selected a sampling period of 18.00h-08.00h. In confirmation of previous findings (Burke et al, 1999) the pattern of expression of the wild-type transgene $(-218 / \mathrm{Luc})$ is characterized by a rhythm with peak values at either the $24.00 \mathrm{~h}$ or $02.00 \mathrm{~h}$ time-points (Fig.2). A similar rhythm of expression was observed for the endogenous Aanat transcript (Fig.2). Comparison of the timecourses generated for these two transcripts obtained by plotting values relative to the daily peak revealed that they were nearly identical, except for a minor difference in the shape of the two curves (Fig.2B). The similar overall pattern and amplitude of the rhythm is consistent with similar rates of transcription of both transcripts and confirms previous studies using a similarly designed construct with a chloramphenicol acetyltransferase (CAT) reporter (Burke et al, 1999).

Analysis of the expression of the mutated transgene $(-218 \mathrm{mEB} / \mathrm{Luc})$ revealed that it exhibited a marked daily rhythm of expression (Fig.2) that was generally similar, albeit not identical to the rhythm of native Aanat mRNA in the same samples. Statistical comparison of the mutant $(-218 \mathrm{mEB} / \mathrm{Luc})$ and wild-type $(-218 / \mathrm{Luc})$ transgene rhythms failed to reveal significant differences in any of the three mutant transgenic lines (Fig.2). The absence of differences in rhythmic gene expression between the mutant and wildtype lines could not be attributed to differences in transgene copy number because the three mutant lines had variable copy numbers and the wild-type line has a copy number that is very similar to the U20 and U42 mutant lines (Fig.1C). These observations indicate that the E-box sequences in intron 1 are not required for rhythmic expression of the gene in the rat pineal gland. 
Tissue specific expression. The issue of issue specific expression was examined using Northern blot analysis (Fig.3). In the wild-type transgenic line (-218/Luc) transgene expression was restricted to the pineal gland and retina; this has been observed previously for the CAT transgenic lines (Burke et al, 1999) and for native Aanat mRNA. However, a different tissue expression pattern was observed in the $-218 \mathrm{mEB} / \mathrm{Luc}$ lines (Fig.3), characterized by significant ectopic expression in the cortex, hypothalamus and spleen. In the cerebral cortex of the U30 line animals, transgene transcript levels were elevated above retinal levels (Fig. 3B). Similar results were observed in a duplicate biological replicate of the U30 line and in duplicate replicates of the U42 line.

Northern analysis of retinal extracts (Fig.3) indicated that the levels of expression of the 218/Luc and the $-218 \mathrm{mEB} /$ Luc transgenes were not markedly different. We also performed in situ hybridization analysis to determine whether there were marked differences in the pattern of transgene expression in the retina. This overall intensity of transgene expression in the $-218 \mathrm{mEB} / \mathrm{Luc}$ and $-218 /$ Luc lines was similar, however, there was a marked change in the pattern of cellular expression characterized by a greater spread across the ONL and increased expression in the INL of the $-218 \mathrm{mEB} / \mathrm{Luc}$ lines. The change in the expression pattern within cell layers of the retina is consistent with the view that the intronic Aanat E-Boxes function to mediate ectopic silencing of the gene.

Comparison of light and dark phase retinal samples indicated that the transgene was rhythmically expressed in the ONL in the $-218 /$ Luc line but not in that of the - 
$218 \mathrm{mEB} /$ Luc lines; however, the extended distribution of the transcript across the ONL precludes clear visualization and objective analysis of rhythmic expression within a subpopulation of ONL cells.

\section{DISCUSSION}

The present study has provided a number of insights into the regulation of rat Aanat gene expression. First, we have discovered that E-box sequences within intron 1 are not required to drive or modulate the physiological circadian expression of the gene in the pineal gland (Baler et al, 1999; Chen \& Baler, 2000; Burke et al, 1999). Second, we have determined that E-box sequences within intron 1 are required for the maintenance of tissue-specific expression because mutation of the +623 E-box and +636 E-box-like sequence in a transgenic context resulted in ectopic expression of the transgene within the brain and retina. These results are entirely consistent with previous studies that indicated a role, firstly, for intron 1 (Burke et al, 1999), and secondly, specifically for the intron 1 E-box in specifying rat Aanat gene expression (Baler et al, 1999; Chen \& Baler, 2000). The findings of the current study provide convincing evidence because they were generated in vivo, in a physiological context.

Our finding that intron 1 E-box mutations do not affect rhythmic pineal expression of Aanat is also consistent with previous studies that found no evidence that Bmall and Clock proteins act through the intron 1 E-box to alter expression in cultured pinealocytes (Chen \& Baler, 2000). The latter result is intriguing because it indicates that this element 
is functionally refractory to these clock proteins in a pinealocyte context where these proteins appear to be expressed (Namahira et al, 1999 Karolczak et al, 2004). In contrast, similar experiments using retinal photoreceptor cells indicate that Bamlland Clock can up-regulate transcription from this element (Chen \& Baler, 2000). This emphasizes both the fundamental differences that exist between the intracellular milieus of these closely related tissues and the importance of cellular context in the analysis of regulatory elements.

The molecular basis of the pineal/retinal difference is unknown but may involve cellspecific factors that possibly interact with elements that flank the E-box sequences (Munoz et al, 2002). The absence of any effect of E-box mutations on rhythmic Aanat expression in the pineal gland is not surprising in light of the large body of evidence that supports the view that the dominant mechanism controlling expression of Aanat in the rodent involves cAMP acting through a CRE-CCAAT complex element in the proximal 5' region of the Aanat gene (Baler et al, 1997; Klein, 2006). The dominant role of a cAMP/CRE pathway in the control of rat pineal Aanat expression appears to contrast, however, with modes of pineal Aanat regulation in other species, for example, the zebrafish Danio rerio where E-box elements do appear to contribute to circadian regulation of the zfaanat2 gene (Appelbaum et al, 2005). Similarly, in the avian pineal gland and retina, the primary mechanism controlling Aanat expression is the Bmal1/Clock interaction with E-box elements; the role that cAMP plays appears to be secondary (Chong et al, 2000; 2003; Iuvone 2005; Klein, 2006). These differences are in 
accordance with known diversity in the organization of the systems that control Aanat among vertebrates (Klein et al, 1997; Klein, 2006).

The transgenic demonstration that E-box sequences act in the specification of rat Aanat gene expression presented here firmly establish a role for these intronic sequences in the control of tissue/celluar specificity in mammals. Previous in vitro analysis of regulatory Aanat intronic sequence addressed the gross contribution of blocks of intron 1 sequence to tissue specificity by measuring relative transcriptional activity in a pituitary cell line (AtT20 cells, Baler et al, 1999). The deletion mutant that approximates most closely to the point mutants examined here $(\Delta 622-1010)$ was found not to have a significant effect on the relative level of expression in these cells. This finding is in accordance with our current in vivo data that does not indicate any up-regulation of pituitary expression in the E-box mutants. In contrast, other deletion mutants in the Baler et al (1999) study, for example $\Delta 523-1563$, exhibited a marked enhancement of pituitary cell expression. This, together with the finding ectopic expression in the E-box mutant lines, suggests that sequences within the Aanat intron contribute differentially to tissue specific repression of ectopic expression. Our results are therefore consistent with the notion that intron $1 \mathrm{E}-$ box sequences contribute to tissue specificity but are not solely responsible for this facet of Aanat gene expression. It is apparent that silencing mechanisms exist in non-pineal tissues to suppress ectopic expression and that these may involve tissue/cell specific factors that interact with different elements within Aanat intron 1. Previous studies by Chen and Baler (2000) have shown that tissue extracts from a number of brain regions 
including cerebral cortex contain factors that bind the intron 1 E-box in vitro. However, the identity and cellular-specificity of these factors is currently unknown.

A role for E-box elements in the maintenance of appropriate pineal-specific expression has also been addressed in the zebrafish model. In this fish species it has been shown that a downstream E-box element is necessary but not sufficient for the maintenance of pineal-specific expression of the zfaanat2 gene in zebrafish (Appelbaum et al, 2004; Appelbaum \& Gothilf, 2006). The results of these transgenic fish experiments suggest, in fact, that the E-box has a dual role in both the suppression of ectopic expression and in the enhancement of pineal expression. The present data from the rat is inconsistent with the latter function because the E-box mutant $(-218 \mathrm{mEB} / \mathrm{Luc})$ transgenic lines did not exhibit a reduction in pineal expression (Fig. 3B). One explanation of this is that the levels of clock genes expressed in the zebrafish pineal are sufficiently high to control gene expression.

Another explanation involves the context within which the E-box exists in the zebrafish Aanat gene, where it is functionally interactive with PCEs within the 3' enhancer (Appelbaum et al, 2004; Appelbaum \& Gothilf, 2006). Our current demonstration of a (partially) conserved role, but different gene location (intronic v 3' flanking sequence) for the Aanat E-box sequences is consistent with current comparative genomic studies that have identified location shuffling of cis-elements in gene orthologues (Sanges et al, 2006). A number of PCEs are located within rat Aanat intron 1 (Fig.1A) but the functional role of these putative regulatory elements either individually, or in association 
with the intronic E-box elements has not been investigated. It is apparent, however, that a possible collective (modular) role of the rat intronic E-box and PCE elements may be cell-type specific, being dependent upon trans-acting and /or epigenetic components. This supposition is based upon the published evidence of differential activities of the intronic E-box in pineal and retinal photoreceptor cells (Chen \& Baler, 2000), and the supportive findings of the present study which show the putative circadian E-box elements in intron 1 of the Aanat gene do not contribute to rhythmic expression in the pineal gland of transgenic rats. Further studies of the functional role of Aanat E-boxes are warranted, particularly given the presence of these sequences within the Aanat gene in representative species across vertebrate evolution (Ensembl: www.ensembl.org).

In conclusion, we have found that E-box elements play a role in the regulation of tissuespecificity of the rat Aanat gene but not pineal rhythmicity. This finding further advances our understanding of E-boxes, important DNA elements that mediate a broad range of regulatory mechanisms following rules that remain poorly defined (Kewley et al, 2004; Munoz et al, 2002). 


\section{ACKNOWLEDGEMENTS}

Support from the Wellcome Trust (AH, DAC) is gratefully acknowledged. This research was also supported in part by the Intramural Research Program of the National Institute of Child Health and Human Development, National Institutes of Health, USA. 


\section{REFERENCES}

Appelbaum, L., Anzulovich, A., Baler, R., Gothilf, Y., 2005. Homeobox-clock protein interaction in zebrafish. A shared mechanism for pineal-specific and circadian gene expression. J. Biol. Chem. 280, 11544-11551.

Appelbaum, L., Gothilf, Y., 2006. Mechanism of pineal-specific gene expression: the role of E-box and photoreceptor conserved elements. Mol. Cell. Endocrinol. 252, 27-33.

Appelbaum, L., Toyama, R., Dawid, I.B., Klein, D.C., Baler, R., Gothilf, Y., 2004. Zebrafish serotonin-N-acetyltransferase-2 gene regulation: pineal-restrictive downstream module contains a functional E-box and three photoreceptor conserved elements. Mol. Endocrinol. 18, 1210-1221.

Baler, R., Covington, S., Klein, D.C., 1997. The rat arylalkylamine N-acetyltransferase gene promoter. cAMP activation via a cAMP-responsive element-CCAAT complex. J. Biol. Chem. $272,6979-6985$

Baler, R., Covington, S., Klein, D.C., 1999. Rat arylalkylamine N-acetyltransferase gene: upstream and intronic components of a bipartite promoter. Biol. Cell. 91, 699-705. 
Burke, Z.D., Wells,T., Carter,D.A., Klein,D.C., Baler,R., 1999. Genetic targeting: The serotonin N-acetyltransferase promoter imparts circadian expression selectively in the pineal gland and retina. J.Neurochem. 73, 1343-1349.

Chen,W., Baler, R., 2000. The rat arylalkylamine N-acetyltransferase E-box: differential use in master vs. a slave oscillator. Mol. Brain Res. 81, 43-50.

Chong, N.W., Bernard, M., Klein, D.C., 2000. Characterization of the chicken serotonin $\mathrm{N}$-acetyltransferase gene. Activation via clock gene heterodimer/E box interaction. J. Biol. Chem. 275, 32991-32998.

Chong, N.W., Chaurasia, S. S., Haque, R., Klein, D.C., Iuvone, P.M., 2003. Temporal-spatial characterization of chicken clock genes: circadian expression in retina, pineal gland, and peripheral tissues. J. Neurochem. 85. 851-860.

Coon, S., Roseboom, P.H., Baler, R., Weller, J.L., Namboodiri, M.A.A., Koonin, E.V., Klein,D.C.,1995. Pineal serotonin $N$-acetyltransferase: expression cloning and molecular analysis. Science 270, 1681-1683

Glowinski, J., Iversen, L.L., 1966. Regional studies of catecholamines in the rat brain. I. The disposition of $[3 \mathrm{H}]$ norepinephrine, $[3 \mathrm{H}]$ dopamine and $[3 \mathrm{H}]$ dopa in various regions of the brain. J. Neurochem. 13, 655-669. 
Iuvone, P.M., Tosini, G., Pozdeyev. N., Haque, R., Klein, D.C., Chaurasia, S.S., 2005. Circadian clocks, clock networks, arylalkylamine N-acetyltransferase, and melatonin in the retina. Prog. Retin. Eye Res. 24, 433-456.

Jin, X., Shearman, L.P., Weaver, D.R., Zylka, M.J., de Vries, G.J., Reppert, S.M., 1999. A molecular mechanism regulating rhythmic output from the suprachiasmatic circadian clock. Cell 96, 57-68.

Karolczak, M., Burbach, G.J., Sties, G., Korf, H.W., Stehle, J.H., 2004. Clock gene mRNA and protein rhythms in the pineal gland of mice. Eur. J. Neurosci.19, 3382-3388.

Kewley, R.J., Whitelaw, M.L., Chapman-Smith, A., 2004. The mammalian basic helixloop-helix/PAS family of transcriptional regulators. Int. J. Biochem. Cell Biol. 36, 189204.

Klein, D.C., 2006. Arylalkylamine N-acetyltransferase: "The timezyme"., 2006. J. Biol. Chem. http://www.jbc.org/cgi/doi/10.1074/jbc.

Klein, D., Coon, S., Roseboom, P.H, Weller, J.L., Bernard, M., Gastel, J.A., Zatz, M., Iuvone, P.M., Rodriguez, I.R., Begay, V., Falcon, J., Cahill, G.M., Cassone, V.M., Baler,R., 1997. The melatonin rhythm-generating enzyme: molecular regulation of serotonin $\mathrm{N}$-acetyltransferase in the pineal gland. Recent. Prog. Horm. Res. 52, 307-337. 
Maronde, E., Pfeffer, M., Olcese, J., Molina, C.A., Schlotter, F., Dehghani, F., Korf, H.W., Stehle, J.H., 1999. Transcription factors in neuroendocrine regulation: rhythmic changes in pCREB and ICER levels frame melatonin synthesis. J. Neurosci. 19, 3326-3336

Munoz, E., Brewer, M., Baler, R., 2002. Circadian Transcription. Thinking outside the E-Box. J. Biol. Chem. 277, 36009-36017.

Namahira,H., Honma,S., Abe,H., Tanahashi,Y., Ikeda,M., Honma,K., 1999. Daily variation and light responsiveness of mammalian clock genes, Clock and Bmal1, in the pineal body and different areas of brain in rats. Neurosci. Lett. 267, 69-72.

Reppert, S.M., Weaver, D.R., 2002. Coordination of circadian timing in mammals. Nature 418, 935-941.

Roseboom, P.H., Coon, S.L., Baler, R., McCune, S.K., Weller, J.L., Klein, D.C. , 1996. Melatonin synthesis: analysis of the more than 150-fold nocturnal increase in serotonin $\mathrm{N}$-acetyltransferase messenger ribonucleic acid in the rat pineal gland. Endocr. 137. 3033-3045.

Roseboom, P.H., Klein, D.C., 1995. Norepinephrine stimulation of pineal cyclic AMP response element-binding protein phosphorylation: primary role of a beta-adrenergic receptor/cyclic AMP mechanism. Mol. Pharmacol. 47, 439-449 
Sanges, R., Kalmar, E., Claudiani, P., D'Amato, M., Muller, F., Stupka, E., 2006. Shuffling of cis-regulatory elements is a pervasive feature of the vertebrate lineage. Genome Biol. 7, R56

Smith, M., Burke, Z., Humphries, A., Wells, T., Klein, D., Carter, D., Baler, R. (2001)

Tissue-specific transgenic knock-down of Fos related antigen-2 (Fra-2) expression mediated by a dominant negative Fra-2. Mol. Cell. Biol. 21, 3704-3713. 


\section{FIGURE LEGENDS}

Fig.1. Transgene design and transgenic line genotype. A. Schematic representation of the rat Aanat gene showing the four exons (boxes) and three introns. The positions of selected regulatory elements are indicated by vertical lines. $\mathrm{P}=$ photoreceptor conserved element. B. Schematic representation of the two transgene constructs: a wild-type transgene (-218/LUC) composed of 218 bases of 5' Aanat promoter sequence together with the next 1786 bases of downstream sequence that includes exon 1 and partial exon 2 sequence and the intervening intron 1, linked to the luciferase reporter gene; a mutant transgene (-218mEB/LUC) that includes mutations in the Aanat intronic E-box and Ebox like sequences. C. Phosphor screen image of a representative Southern blot of PstIdigested genomic DNA extracted from each of the four transgenic lines (U20, U30, U42, W21) and two wild-type rats (wt). Note the presence of a diagnostic $\sim 3 \mathrm{~kb}$ transgenespecifc band (arrow) that is present at different intensities according to the transgene copy number of individual lines.

Fig.2. Rhythms of transgene expression in rats transgenic for either a wild-type (218/Luc) or mutant (-218mEB/LUC) Aanat promoter-reporter transgene. Total RNA was extracted from pineal glands of rats killed at different times of the circadian cycle. Levels of transgene (luciferase reporter gene, Luc) and endogenous Aanat transcripts were quantified with a ribonuclease protection assay (RPA, see text), and compared with levels of a control transcript (GAPDH). A. Representative phosphor screen images of protected 
RPA products (sizes in nucleotides, nt) in samples taken across the circadian cycle. $\mathbf{B}$. Graphical representation of summated RPA data showing the levels of Luc and Aanat transcripts plotted as a percentage of peak values. Each data point is mean \pm S.E.M. of three independent pools of pineal glands. Statistical analysis of wild-type transgene transcript (Luc) abundance across the cycle revealed a highly significant between group difference ( $\mathrm{F}=775.119 ; \mathrm{p}<0.0001,1$-way ANOVA). Comparison of the mutant ($218 \mathrm{mEB} / \mathrm{Luc})$ and wild-type (-218/Luc) transgene rhythms by Area under curve measurement and Student's t test did not reveal any significant differences $(p=0.132$ for the U30 line shown in this Figure).

Fig.3. Tissue distribution of transgene expression. Northern blot of transgene and endogenous Aanat transcripts in rats transgenic for either a wild-type (-218/Luc) or mutant (-218mEB/LUC) Aanat promoter-reporter transgene. Total RNA (8mg/lane) was extracted from pineal glands (pool of two) and other tissues (Pit, pituitary gland; Hyp, hypothalamus; Cor, cerebral cortex; Ret, retina; Adr, adrenal gland; Ov, ovary; Kid, kidney; Liv, Liver; Spl, spleen; Ht, heart) of rats killed at 24.00h and subjected to Northern analysis as described in the text. Blots were probed with cDNA probes specific for the transgene transcript (Luc) or Aanat transcript, and re-probed with an $18 \mathrm{~S}$ ribosomal RNA probe. A. Representative phosphor screen images of Northern blots conducted on the W21 (-218/Luc) and U30 (-218mEB/LUC) lines are shown., and similar results were obtained in a second, independent line of MUT-Tg rats. Note that transgene expression is restricted to the pineal gland and retina in the $-218 /$ Luc line, but that expression is relaxed in the $-218 \mathrm{mEB} / \mathrm{LUC}$ line with transgene expression being 
clearly detected in both the hypothalamic and cortical regions of the brain. B. Graphical representation of the data in (A). Levels of the transgene transcript (Luc) were quantified by densitometry, corrected against the corresponding level of 18S RNA and expressed as a percentage of the level in retina. Note that the pineal gland values are presented above the corresponding histograms.

Fig.4. In situ hybridization analysis of transgene expression in the retina of rats transgenic for either a wild-type (-218/Luc) or mutant (-218mEB/Luc) Aanat promoterreporter transgene. Whole eyes were obtained from rats killed at either 18.00h (CT18) or 24.00h (CT24) and frozen $(12 \mathrm{~mm})$ transverse sections were processed for nonradioactive in situ hybridization analysis using digoxigenin-labelled, sense and antisense RNA probes as described in the text. A. Haematoxylin and Eosin-stained section of the retina showing the main cellular layers: OS (outer segments), IS (inner segments) and ONL (outer nuclear layer) of the photoreceptor cells; INL (inner nuclear layer), and GCL (ganglion cell layer). B. Representative image of a retinal section from a -218/Luc rat probed with a sense orientation transgene probe. Note the detection of a minor level of signal that is apparent in the GCL. The scale bar, which is representative for all images, is $20 \mu \mathrm{m}$. C. Representative images of retinal sections from $-218 / \mathrm{Luc}$ and $-218 \mathrm{mEB} / \mathrm{Luc}$ rats probed with an antisense transgene probe. Note that transgene-positive cells are primarily located in the outer (upper) zone of the ONL in the $-218 / \mathrm{Luc}$ line, and that the signal intensity is greater at CT24 compared with CT18. In contrast, in the $-218 \mathrm{mEB} / \mathrm{Luc}$ line transgene-positive cells are found in a wider distribution across the ONL, and in addition, 
there is significant expression within cells of the INL. A consistent temporal difference in expression was not detected in the $218 \mathrm{mEB} / \mathrm{Luc}$ retina. 
TABLE 1: Oligonucleotides used in construction of qRPA probes

\begin{tabular}{|r|l|l|l|}
\hline \multicolumn{1}{|c|}{ Gene } & Acc. No. & Position & \multicolumn{1}{c|}{ Sequence } \\
\hline GAPDH s & NM_017008 & $585-611$ & tgtggatggccctctggaaagctgtggcgtgatg \\
\hline as & & & ttaacatcacgccacagctttccagaggggccatccacaat \\
\hline AANAT s & NM_012818 & $399-433$ & atgagatccggcacttcctcaccetgtgtccagag \\
\hline as & & & ttaactctggacacagggtgaggaagtgccggatctcatat \\
\hline LUC s & U03687 & $1084-1118$ & ggagttgcagttgcgcccgcgaacgacatttataa \\
\hline as & & & ttaattataaatgtcgttcgcgggc gcaactgcaactccat \\
\hline
\end{tabular}

Oligonucleotide sequences are 5' - 3'.

LUC, luciferase; s, sense; as, antisense. 
FIGURE 1

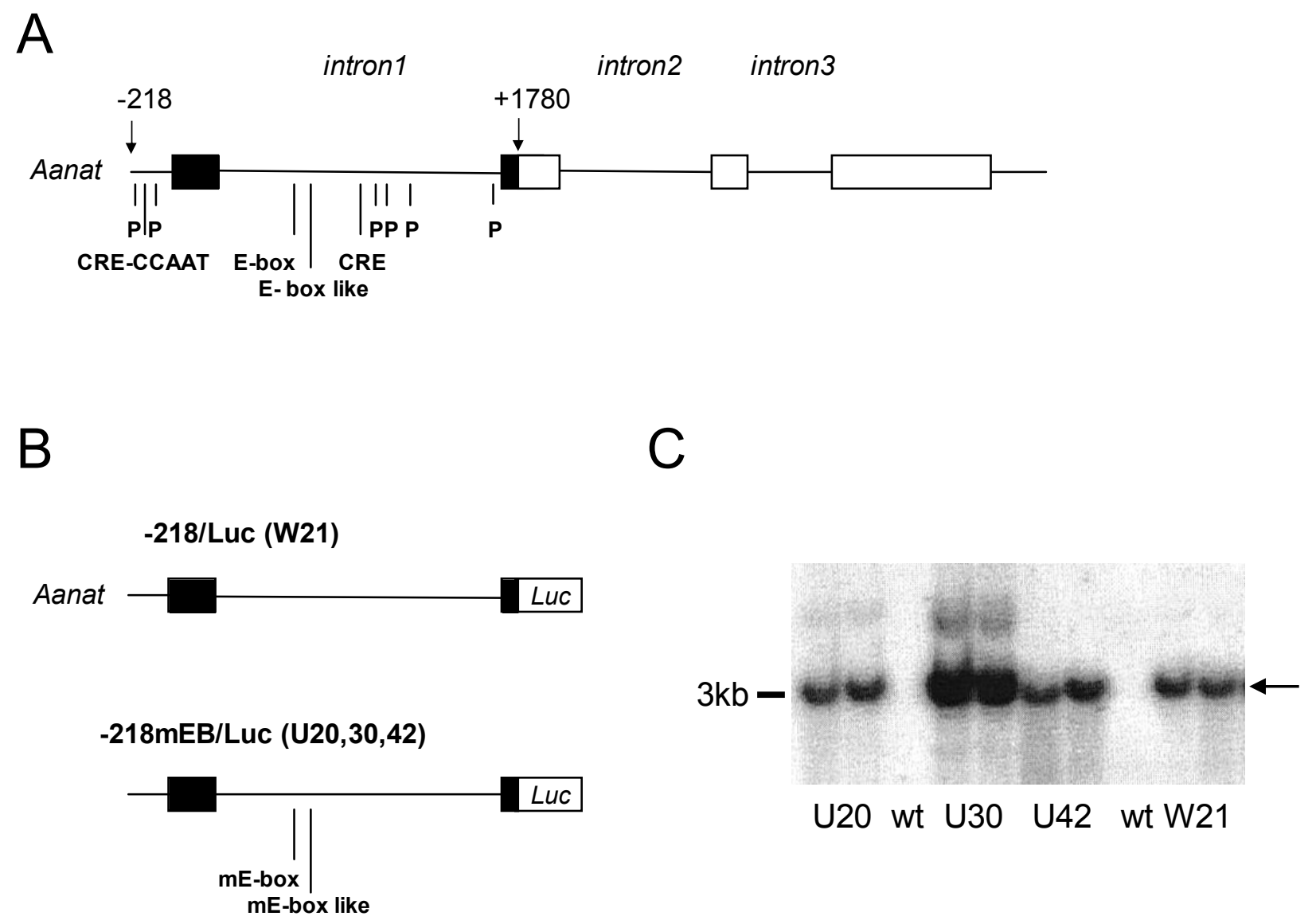




\section{FIGURE 2}

A

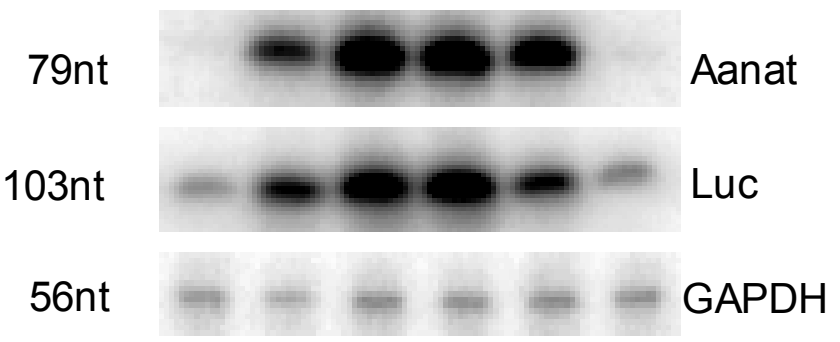

$\begin{array}{llllll}18 & 22 & 24 & 02 & 04 & 08 \mathrm{~h}\end{array}$

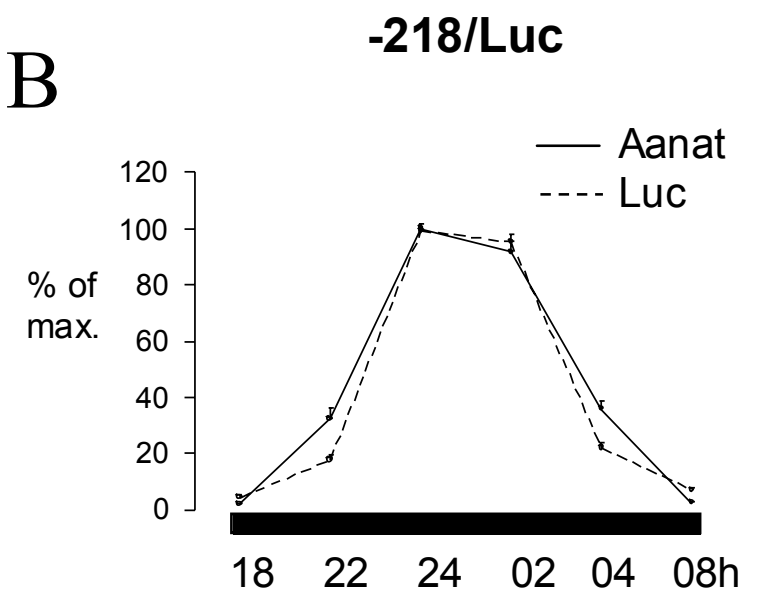

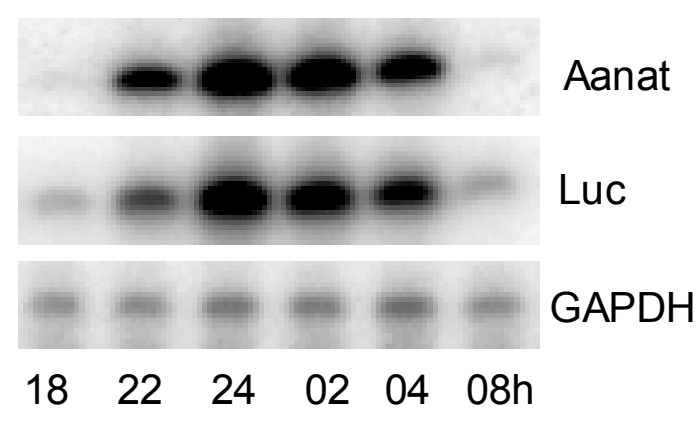

$-218 m E B / L u c$

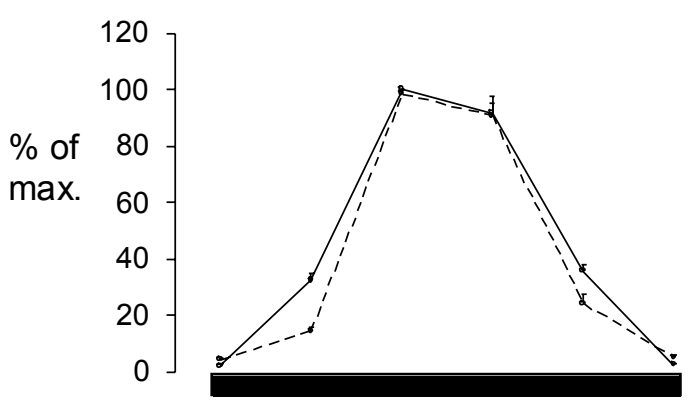

$\begin{array}{llllll}18 & 22 & 24 & 02 & 04 & 08 h\end{array}$ 


\section{FIGURE 3}

A

$-218 /$ Luc

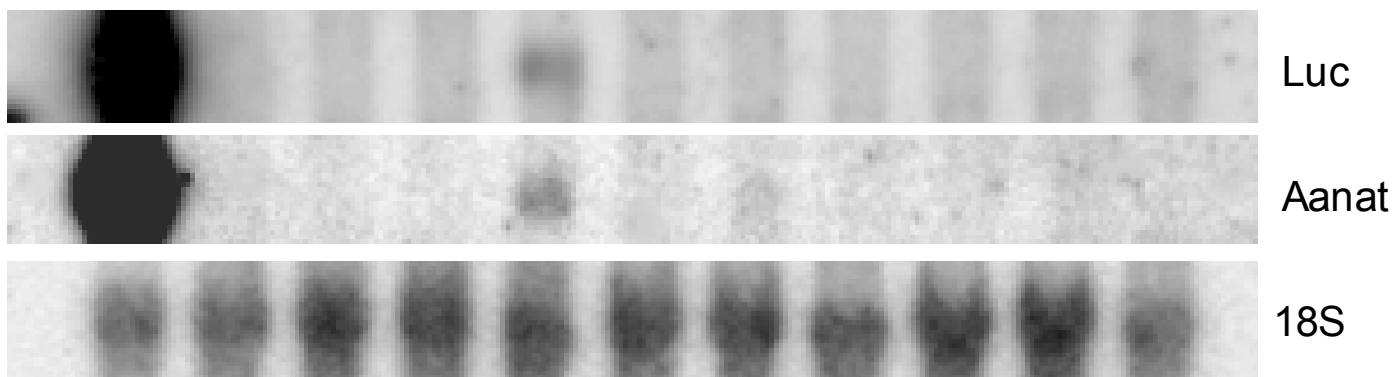

Pin Pit Hyp Cor Ret Adr Ov Kid Liv Spl Ht

$-218 m E B / L u c$

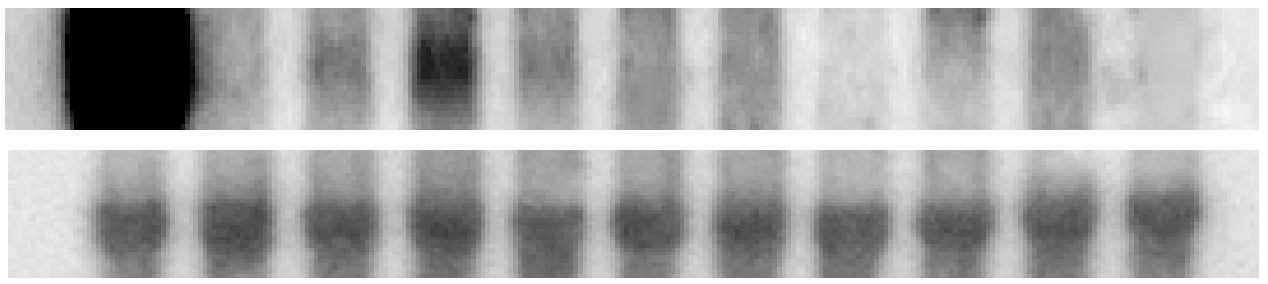

Luc

Pin Pit Hyp Cor Ret Adr Ov Kid Liv Spl Ht

B

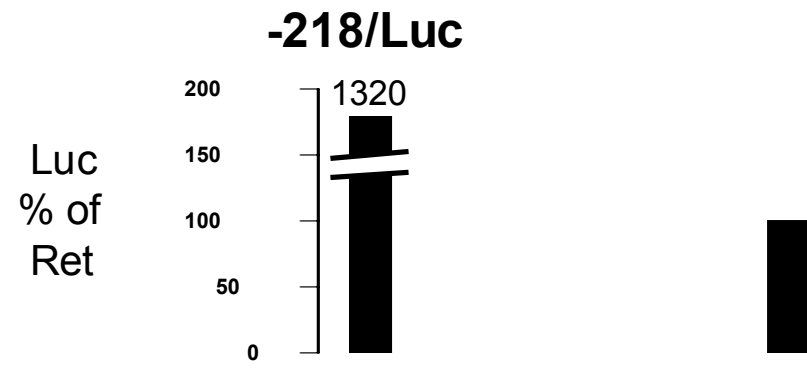

Pin Pit Hyp Cor Ret Adr Ov Kid Liv Spl Ht

\section{$-218 \mathrm{mEB} /$ Luc}

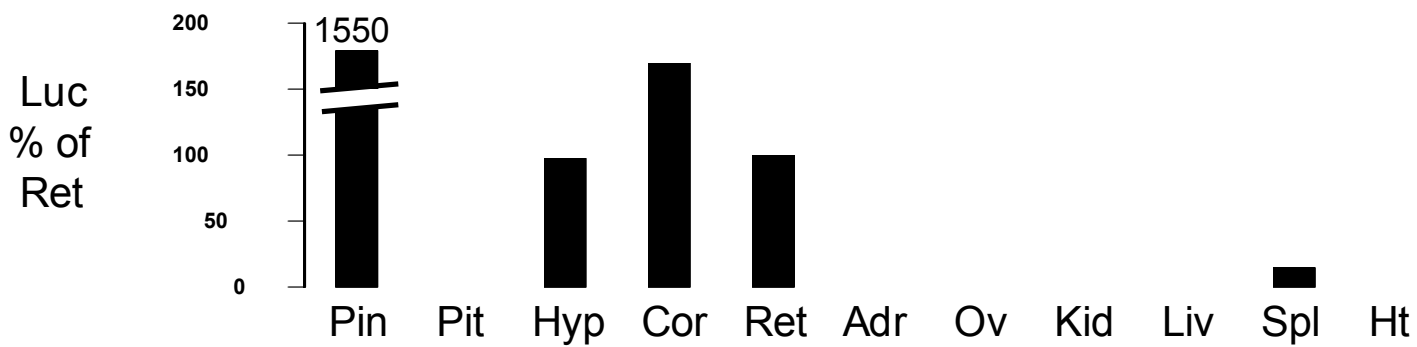


FIGURE 4

A
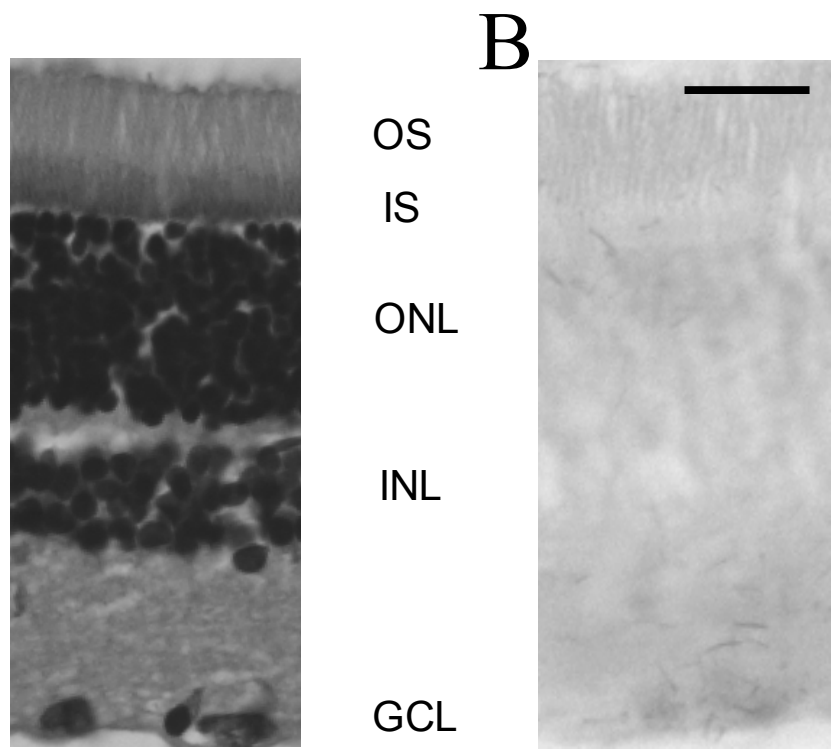

C

$-218 /$ Luc retina
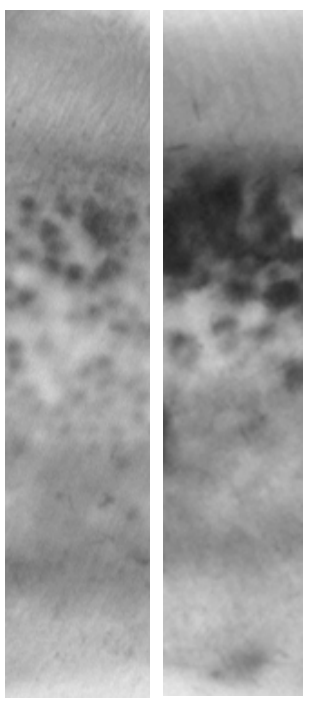

Os

IS

ONL

$-218 m E B / L u c$ retina

CT18 CT24

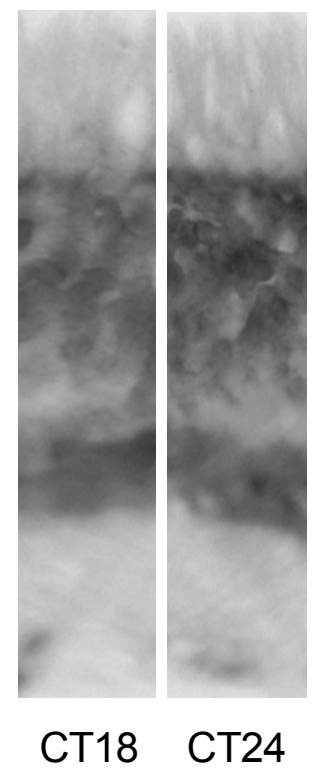

